\title{
BMJ Open Symptom presentation of SARS-CoV-2- positive and negative patients: a nested case-control study among patients calling the emergency medical service and medical helpline
}

\author{
Amalie Lykkemark Moeller (D) , ${ }^{1}$ Elisabeth Helen Anna Mills (D) , \\ Helle Collatz Christensen, ${ }^{3,4}$ Filip Gnesin, ${ }^{1}$ \\ Stig Nikolaj Fasmer Nikolaj Blomberg (D) , ${ }^{4}$ Nertila Zylyftari, ${ }^{5}$ Britta Jensen, ${ }^{6}$ \\ Kristian Bundgaard Ringgren, ${ }^{7}$ Marcella Ditte Broccia, ${ }^{1,8}$ Henrik Bøggild, ${ }^{6}$ \\ Christian Torp-Pedersen ${ }^{1,7,9}$
}

To cite: Moeller AL, Mills EHA, Collatz Christensen $\mathrm{H}$, et al. Symptom presentation of SARSCoV-2-positive and negative patients: a nested case-control study among patients calling the emergency medical service and medical helpline. BMJ Open 2021;11:e044208. doi:10.1136/ bmjopen-2020-044208

- Prepublication history and additional supplemental material for this paper are available online. To view these files, please visit the journal online (http://dx.doi.org/10.1136/ bmjopen-2020-044208).

Received 27 August 2020 Accepted 09 May 2021

D) Check for updates

(c) Author(s) (or their employer(s)) 2021. Re-use permitted under CC BY-NC. No commercial re-use. See rights and permissions. Published by BMJ.

For numbered affiliations see end of article.

\section{Correspondence to} Ms Amalie Lykkemark Moeller; amalie.lykkemark.moeller@ regionh.dk

\section{ABSTRACT}

Objective Currently effective symptom-based screening of patients suspected of COVID-19 is limited. We aimed to investigate age-related differences in symptom presentations of patients tested positive and negative for SARS-CoV-2.

Design

Setting Calls to the medical helpline (1-8-1-3) and emergency number (1-1-2) in Copenhagen, Denmark. At both medical services all calls are recorded.

Participants We included calls for patients who called for help/guidance at the medical helpline or emergency number prior to receiving a test for SARS-CoV-2 between April $1^{\text {st }}$ and $20^{\text {th }} 2020$ (8423 patients). Among these calls, we randomly sampled recorded calls from 350 patients who later tested positive and 250 patients tested negative and registered symptoms described in the call.

Outcome

Results After exclusions, 544 calls (312 SARS-CoV-2 positive and 232 negative) were included in the analysis. Fever and cough remained the two most common of COVID-19 symptoms across all age groups and approximately $42 \%$ of SARS-CoV- 2 positive and $20 \%$ of negative presented with both fever and cough. Symptoms including nasal congestion, irritation/pain in throat, muscle/joint pain, loss of taste and smell, and headache were common symptoms of COVID-19 for patients younger than 60 years; whereas loss of appetite and feeling unwell were more commonly seen among patients over 60 years. Headache and loss of taste and smell were rare symptoms of COVID-19 among patients over 60 years.

Conclusion Our study identified age-related differences in symptom presentations of SARS-CoV-2-positive patients calling for help or medical advice. The specific symptoms of loss of smell or taste almost exclusively reported by patients younger than 60 years. Differences in symptom presentation across age groups must be considered when screening for COVID-19.
Strengths and limitations of this study

- This is the first study reporting symptoms of COVID-19 as they are presented in medical telephone consultations.

- We investigated the presence of 36 symptoms and reported variations in symptom presentations across age groups.

- By randomly sampling calls to this study from SARS-CoV-2-tested individuals calling the 24-hour medical helpline and emergency medical services, our study included patients from all ages with high generalisability to the general population.

- Information of patient characteristics, including comorbidities, socioeconomic factors, ethnicity and living condition, could have improved the analysis.

\section{INTRODUCTION}

COVID-19 has been classified as a pandemic since 11 March 2020. Politicians worldwide have initiated serious actions in order to hinder spread of the disease and retain sufficient healthcare capacity. ${ }^{1}$ Countries have increased demand for testing and protection equipment, which currently is exceeding production capacity. ${ }^{2}$ The scarce access to resources results in an urgent need to prioritise patients sent to the emergency departments and testing facilities. ${ }^{2}$ Effective symptom-based screening for COVID-19 is difficult: common influenza-like symptoms of fever, cough and fatigue have been reported by patients with COVID-19 $9^{3-5}$; however, these symptoms are experienced by up to $20 \%$ of a population during a winter season. ${ }^{6}$ Other symptoms have been found to include myalgia, headache, sore throat, runny nose, 
diarrhoea, shortness of breath, nausea, vomiting, and loss of smell and taste. ${ }^{3-5} 7$

As countries worldwide rolled out full or partial societal lockdown, people were encouraged mostly to call local healthcare services rather than physical visits, and telephone and video consultations have in many places replaced the physical encounter with patients placing new demands of not only which symptoms are predictive of COVID-19 but also knowledge of how symptoms are communicated.

Although some studies have investigated the symptoms of COVID-19, little attention has been devoted to investigating symptoms as they are described by patients or their relatives/caregivers in telephone consultations. Additionally, it remains unclear whether the described symptoms are common among both young and elderly patients, as none of the previously mentioned studies have reported symptoms according to age groups. In order to improve healthcare professionals' possibilities to screen patients for COVID-19 in telephone consultations, we need to improve knowledge of how patients across ages present their symptoms. We aimed to describe differences in symptom presentation across age groups of patients calling for medical advice or help prior to receiving a test for SARS-CoV-2.

\section{METHODS}

To investigate the symptom presentation of SARS-CoV2-positive and negative patients, we have systematically listened and recorded symptoms described by patients calling for help and guidance at the 24-hour medical helpline (1813-medical helpline) and 1-1-2 emergency number (equivalent to 9-1-1) in Copenhagen, Denmark, prior to a SARS-CoV-2 swab test. The study is conducted as a nested case-control in accordance with the STrengthening the Reporting of OBservational studies in Epidemiology statement.

\section{Setting}

In Denmark, the healthcare system is public and taxfunded, and most medical services including calling for medical help/advice, hospital treatment and SARS-CoV-2 testing are free of charge. Between 1 April and 20 April, symptomatic patients were eligible for testing and could get directly referred by either contacting their general practitioner or by calling the regional 24-hour medical helpline (1813-medical helpline). Additionally, patients either hospitalised or undergoing surgery were tested if they were symptomatic. In the Capital Region, Denmark, inhabitants should, and most often do, contact the 1813medical helpline, 1-1-2 emergency number or a general practitioner to get referred to an emergency department. $^{8}$ The 1813-medical helpline and the 1-1-2 emergency number is a combined medical service co-located at one facility, the Copenhagen Emergency Medical Service (EMS), serving 1.8 million citizens. ${ }^{8}$ Citizens with non-urgent medical conditions should contact their general practitioner during opening hours on working days or dial 1-8-1-3 to reach the 24-hour medical helpline, where healthcare professionals including nurses and doctors provide medical guidance, refer to emergency departments and dispatch ambulances if necessary. In case of an emergency, citizens should call the emergency number 1-1-2. Due to extraordinary workload caused by COVID-19, the 1813-medical helpline opened a COVID-19 hotline. When dialling 1-8-1-3, patients calling regarding COVID-19 were referred to the COVID-19 hotline, whereas patients calling regarding other matters remained in queue for the 1813-medical helpline. Both patients calling the COVID-19 hotline and directly to the 1813-medical helpline could be referred to testing.

All symptoms indicative of infection could result in referral to a SARS-CoV-2 test. These symptoms included fever, cough, breathing difficulties, sore throat, headache and muscle pain, but was not limited to these as health professionals at the 1813-medical helpline, COVID-19 hotline, general practice and hospitals should assess the patient and refer to test accordingly. At the COVID-19 hotline and 1813-medical helpline, patients were typically questioned about age, comorbidity, respiratory distress, fever, cough, headache, muscle pain and diarrhoea. ${ }^{9}$

All calls to the Copenhagen EMS (1813-medical helpline, COVID-19 hotline and 1-1-2 emergency number) are recorded and stored, together with patient information including age, sex, a medical report and the patients' civil registration number (a unique number assigned to all Danish residents). ${ }^{1011}$

\section{SARS-CoV-2 test}

In Denmark, patients suspected of COVID-19 were tested using a PCR test for SARS-CoV-2 with nasopharyngeal swab. Test results were identified from the patient's electronic medical journal and were linked to previous calls to the Copenhagen EMS using the civil registration number. The majority of tests for SARS-CoV-2 have been carried out at hospitals and a minority at the general practitioners, for this article only hospital tests are included.

\section{Symptom presentation}

All researchers collected data on symptoms by listening to calls and recording information in a survey guide. The survey guide included questions on the identity of the caller, purpose of the call, symptoms, and possible risk behaviour including recent travel and contact with SARSCoV-2-positive individuals. Tic boxes were used to confirm presence of specific symptoms and a text box was added to allow for description of possible novel symptoms. The original survey in Danish and an English translation are available in the online supplemental material 1.

The survey was developed and tested in two rounds prior to the data collection. All researchers listened to the same 10 randomly selected test calls in each round. We evaluated inter-rater variability using free-marginal multirater kappa for each test round. ${ }^{12}$ The average kappa value was estimated to be higher than 0.8 in both tests. 


\section{Population}

Between 1 April and 20 April 2020, we identified all calls to the 1813-medical helpline and 1-1-2 emergency number for individuals tested for SARS-CoV-2 up to 48 hours after the call resulting in 14401 calls from 8423 patients.

After exclusion of inconclusive test results, we identified the last call for each patient and randomly sampled 600 patients (350 tested SARS-CoV-2 positive and 250 negative). This would provide the study with a power of $90 \%$ to detect differences in symptom prevalence of $10 \%$ between the two groups with a $5 \%$ significance level and an enrolment rate of 1.5. Calls regarding following matters were excluded: (1) calls not concerning the patient's condition, for example, patients calling to ask about test results or concerning previously requested transports; (2) calls regarding childbirth, assaults, traffic

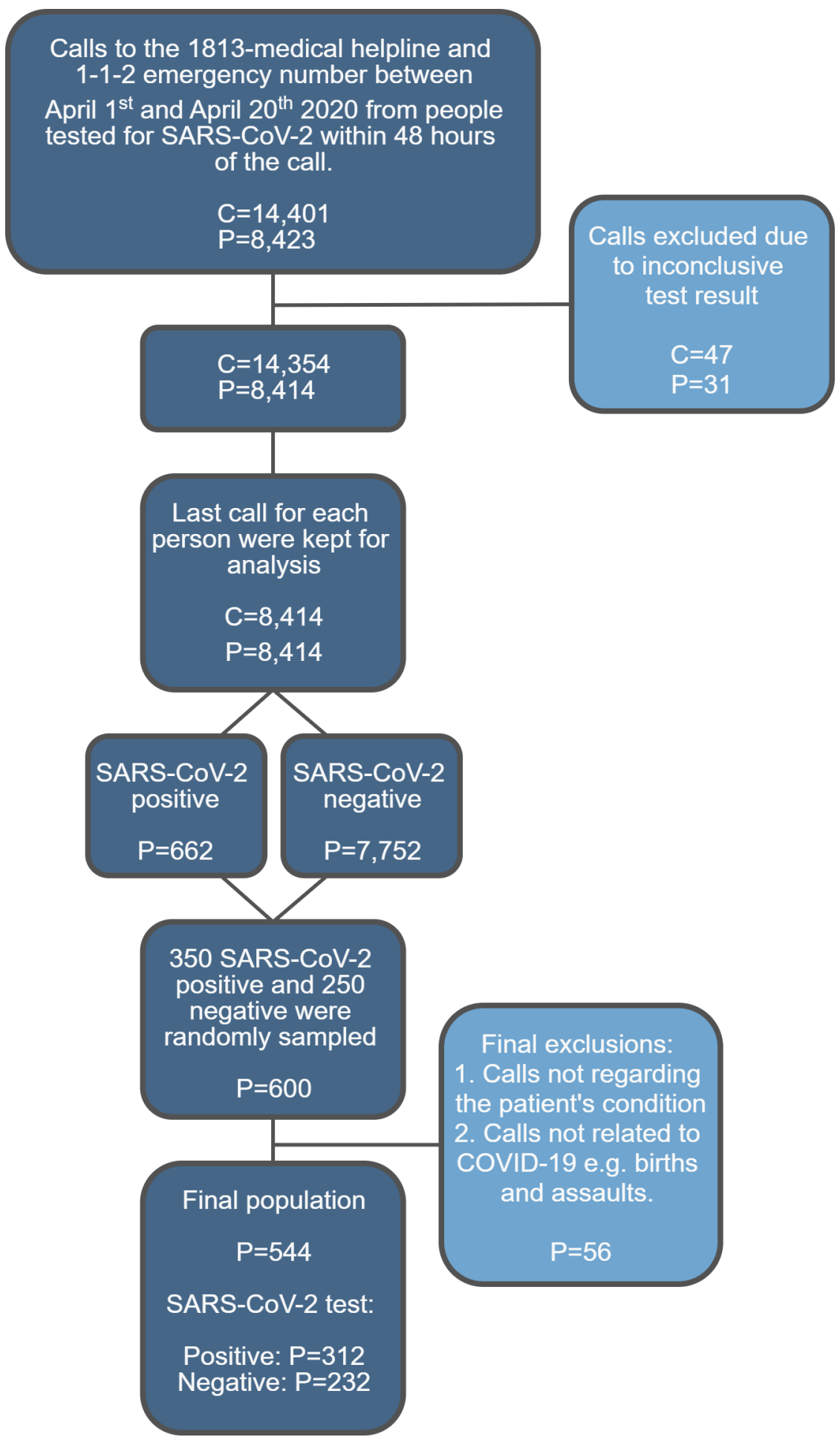

Figure 1 Flow chart describing the selection of calls for SARS-CoV-2-positive and negative patients. C=number of calls; $\mathrm{P}=$ number of people. 
Table 1 Characteristics of patients tested SARS-CoV-2 positive and negative

\begin{tabular}{|c|c|c|c|}
\hline & SARS-CoV-2 positive & SARS-CoV-2 negative & $P$ value \\
\hline & 312 & 232 & \\
\hline Contact with SARS-CoV-2 positive* & $100(32.1)$ & $19(8.2)$ & $<0.001$ \\
\hline Sex (male) & $151(48.4)$ & $93(40.1)$ & 0.056 \\
\hline Age mean (SD) & $51(22.42)$ & $43(24.04)$ & $<0.001$ \\
\hline Under 36 & $89(28.5)$ & $89(38.4)$ & \\
\hline $36-60$ & $116(37.2)$ & $87(37.5)$ & \\
\hline Over 60 & $107(34.3)$ & $56(24.1)$ & \\
\hline Non-emergency calls (1813-medical helpline) & $292(93.6)$ & $212(91.4)$ & 0.407 \\
\hline
\end{tabular}

$\mathrm{N}=544$.

*All patients tested positive for SARS-CoV-2 were advised to quarantine until 48 hours after end of symptoms. Patients can infect others with SARS-CoV-2 days before developing symptoms themselves while they are still unaware of their infection. ${ }^{19}$

accidents or similar obviously not related to COVID-19. Such calls might appear in the random sample as patients who had or developed symptoms during hospitalisation were tested to minimise in-hospital transmission of COVID-19.

\section{Analysis}

Data were collected using Research Electronic Data Capture, which is a secure, web-based software platform designed to support data capture for research studies. ${ }^{13}{ }^{14}$ Prior to analysis, symptoms described in the free text option were classified into one of the pre-existing categories when possible.

The proportion of SARS-CoV-2 positive and negative that presented with each symptom was estimated. $\mathrm{P}$ values with $5 \%$ significance level were estimated using F-test for continuous variables and Fisher's exact test for categorical variables. The proportion of SARS-CoV-2 positive and negative presenting with each symptom was also estimated in three age groups $(0-35,36-60$ and $>60$ years $)$ and in five age groups for sensitivity analysis $(0-17,18-35$, $36-50,51-70$ and $>70$ years). Venn diagrams were used to illustrate the prevalence of combinations of the three most common symptoms in three age groups.

Patients could receive a SARS-CoV-2 test performed by being either referred to a test centre or admitted to a hospital. Therefore, we have possibly included patients calling the Copenhagen EMS for conditions unrelated to COVID-19, for example, cardiovascular disease, who were subsequently tested for SARS-CoV-2 to minimise risk of in-hospital spread. We expected that these patients were less likely to have COVID-19 and more likely to present with chest pain and trauma. To assess whether this biased our results, we investigated the prevalence of the symptoms in the subset of patients suspected of COVID-19 by the healthcare personnel at the Copenhagen EMS, defined as calls where a note of COVID-19 were added to the electronic medical report at the Copenhagen EMS, as we expect that these patients were tested due to suspicion of COVID-19.

Data management and analysis of data were carried out in $\mathrm{R}$ version 3.6.1. ${ }^{15}$

\section{RESULTS}

From 1 April to 20 April 2020, we identified 14401 calls to the 1-1-2 emergency number and 1813-medical helpline from people tested for SARS-CoV-2 within 48 hours of the call. Inconclusive test results were excluded (47 calls from 31 individuals) and only the last call prior to a test was kept resulting in 8414 calls (662 SARS-CoV-2 positive and 7752 negative) (see flow chart in figure 1). We randomly sampled calls from 350 SARS-CoV-2 positive and 250 negative and recorded their symptoms. After exclusion of 56 calls (38 SARS-CoV-2 positive and 18 SARS-CoV-2 negative), 312 calls from SARS-CoV-2-positive and 232 calls from SARS-CoV-2-negative patients were included for analysis (figure 1).

Patients tested positive for SARS-CoV-2 were on average older compared with those tested negative (mean age 51 vs 43 ), and more often men ( $48.4 \%$ vs $40.1 \%$ ) (table 1 ). Among patients tested SARS-CoV-2 positive, $32.1 \%$ had been in contact with an infected individual. This was only true for $8.2 \%$ of those tested negative (table 1 ).

In table 2 the symptoms are presented stratified by SARS-CoV-2 positive and negative. Among the SARS-CoV-2 positive, the most common symptoms were fever $(64.4 \%)$, cough $(63.5 \%)$, breathing problems $(38.8 \%)$ followed by headache $(34 \%)$. The most common symptoms of the SARS-CoV-2 negative were cough (49.1\%), fever (44.8\%), breathing problems $(44 \%)$ and irritation/pain in the throat $(40.1 \%)$. Loss of smell and taste was found to be almost exclusively a symptom among the SARS-CoV-2 positive. Chest pain and irritation/pain in the throat were more common among the SARS-CoV-2 negative than the 
Table 2 Symptoms of patients tested SARS-CoV-2 positive and negative

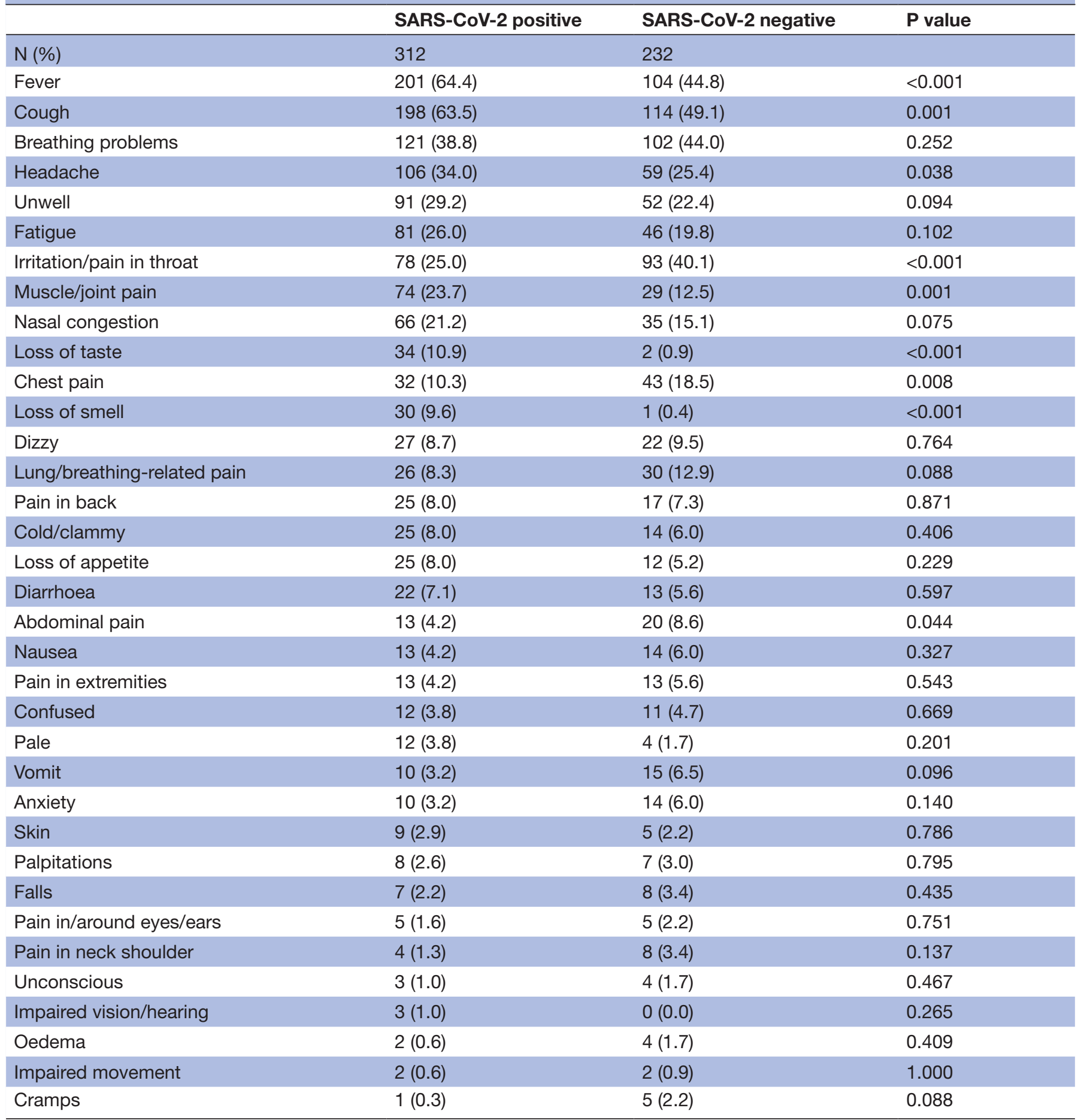

$\mathrm{N}=544$.

positive but neither symptoms were specific for SARSCoV-2-negative individuals.

In figure 2 the symptom presentation of SARS-CoV2-positive and negative patients is shown by age groups (under 36, 36-60 and over 60 years). Among the SARSCoV-2-positive patients younger than 36 years, 36\% presented with nasal congestion, which was twice as common as among the negative $(18 \%)$. Presenting with headache was also common for patients younger than 60 years $(28 \%-34 \%$ of the negative and $43 \%-46 \%$ of the positive), but less common among the patients older than 60 years (9\% of the negative and $14 \%$ of the positive). Similarly, the proportion of both SARS-CoV-2 positive and negative presenting with irritation/pain in the throat decreased with increasing age. Loss of smell and taste was predominantly found among younger SARS-CoV-2positive patients, where $16 \%$ had loss of smell and $16 \%$ had loss of taste. 


\section{Symptom presentation of SARS-CoV-2 positive and negative patients by age group}

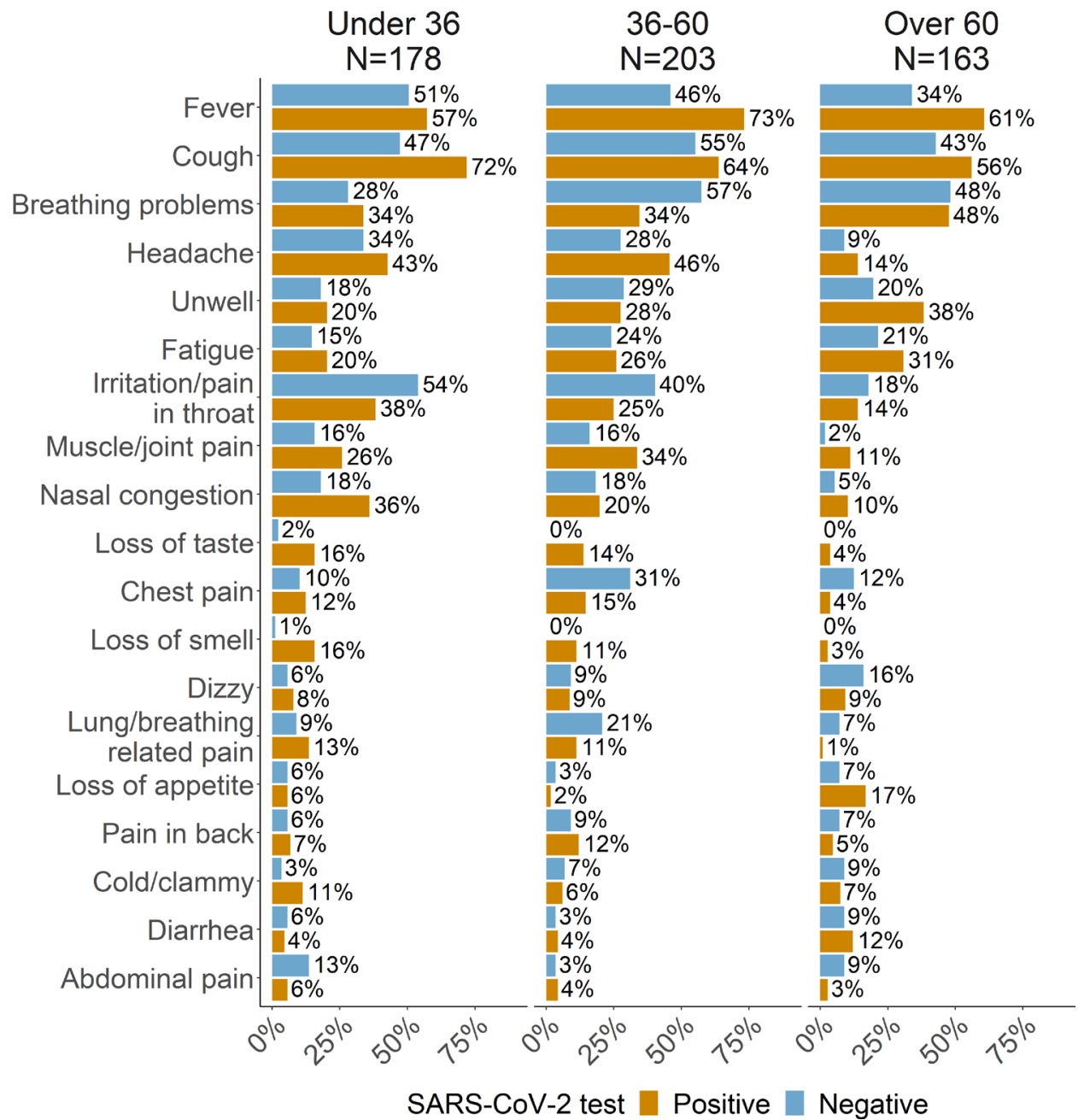

Figure 2 Symptom presentation of SARS-CoV-2 positive and negative by age groups. The figure illustrates the percentage of SARS-CoV-2 positive and negative patients that presented each of the listed symptoms by age groups. Only symptoms experienced by more than 30 patients in total the population $(\mathrm{N}=544)$ were included.

Patients older than 60 years more often presented with loss of appetite (17\% of the SARS-CoV-2 positive and $7 \%$ of the negative). Feeling unwell was also common among the patients over 60 years, especially the SARS-CoV-2 positive (38\% of the SARS-CoV-2 positive vs $20 \%$ of the negative) (figure 2).

In figure 3 the proportion of patients presenting with one or more of the three most common symptoms of that age group is shown for SARS-CoV-2 positive and negative. Presenting with both cough and fever was more common among the SARS-CoV-2 positive (overall: $42 \%$, under 36 : 40\%, 36-60: $47 \%$ and over 60: 38\%) compared with those tested SARS-CoV-2 negative (overall: $20 \%$, under $36: 17 \%$, 36-60: $28 \%$ and over 60: $14 \%$ ). Among patients above age 60 years, $33 \%$ of the SARS-CoV-2 positive presented with breathing problems and fever and 19\% with breathing problems, fever and cough. This symptom combination was less common among SARS-CoV-2-negative patients over 60 years (12\% with fever and breathing problems and 5\% with breathing problems, fever and cough) (figure 3).

\section{Sensitivity analysis}

We investigated the symptom presentation of patients suspected of COVID-19 by the healthcare professionals at the Copenhagen EMS (see online supplemental table $1)$. In the subpopulation, the most common symptoms of the SARS-CoV-2 positive and negative were similar to those found in the total population (SARS-CoV-2 positive: cough $(69.6 \%)$, fever $(69 \%)$ and headache $(38.6 \%)$; SARS-CoV-2 negative: cough $(60.5 \%)$, irritation/pain in throat $(52.4 \%)$ and fever $(49.2 \%))$, although cough was more common among SARS-CoV-2 negative in the subpopulation. The proportion of patients with chest pain was not found to differ between the SARS-CoV-2 positive and negative in this analysis. Loss of smell and taste remained specific among the positive, and similarly 


\section{Combinations of the 3 most common symptoms for each age group (\%) \\ Under 36 \\ SARS-CoV-2 Positive \\ $\mathrm{N}=89$ \\ SARS-CoV-2 Positive \\ $\mathrm{N}=116$ \\ SARS-CoV-2 Positive \\ $\mathrm{N}=107$}
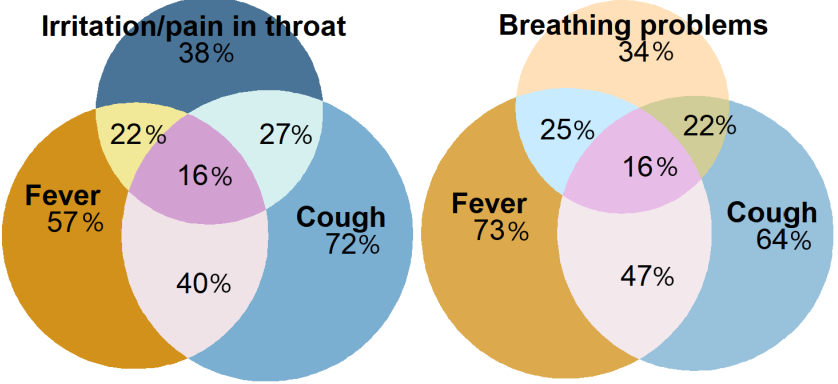

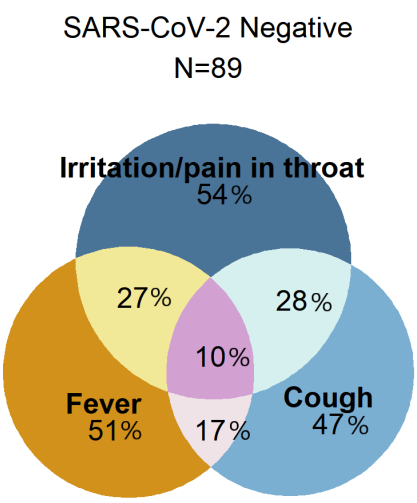

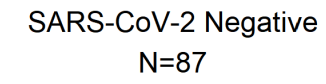

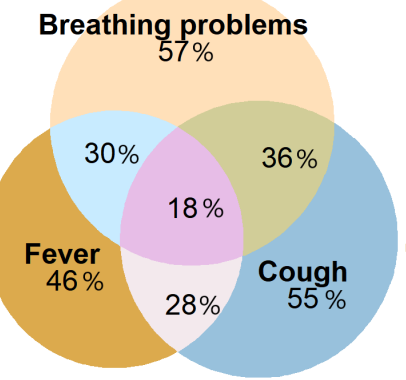

SARS-CoV-2 Negative

$\mathrm{N}=56$

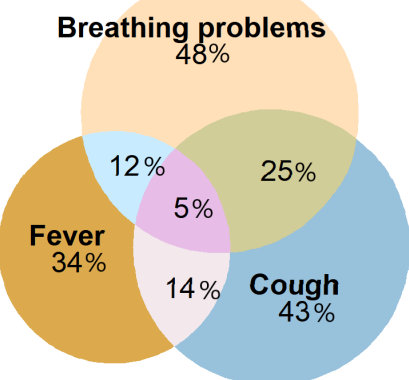

Figure 3 Combinations of the three most common symptoms for each age group by SARS-CoV-2 positive and negative. The percentage represents the prevalence of the given symptom or symptom combination in each subgroup. The area of the bubbles increases with increasing per cent. Overlapping areas indicate a combination of two or three symptoms, and the percentage is the proportion of patients in this subgroup who had this symptom combination. The three most common symptoms were identified for each age group regardless of SARS-CoV-2 test result. For individuals under 36 years, this was cough, fever and irritation/pain in throat; for individuals aged 36-60 and over 60 years, this was fever, cough and breathing problems. $\mathrm{N}=544$ (232 negative and 312 positive).

irritation/pain in the throat remained more common among the negative.

Additionally, we investigated the symptom presentation of the SARS-CoV-2-tested patients across five age groups. Overall, similar patterns were identified when stratifying across five age groups as when stratifying across three age groups (see online supplemental figure 1).

\section{DISCUSSION}

The main finding from this study was that loss of smell and loss of taste were identified as important specific symptoms of COVID-19. The symptom presentations varied across age groups, where symptoms including nasal congestion, muscle/joint pain, loss of taste and smell and headache were common symptoms of COVID-19 for patients younger than 60 years; while loss of appetite and feeling unwell were more common among patients over 60 years.
We found that $10.9 \%$ of SARS-CoV-2 positive had loss of taste and $9.6 \%$ had loss of smell. Previous studies found that among SARS-CoV-2-positive patients-65\% in a British population, $67 \%$ in a US population and $64 \%$ of an Italian population-reported loss of smell or taste. ${ }^{34}$ However, only $2 \%-11 \%$ of SARS-CoV-2-positive individuals in the Icelandic population reported these symptoms. ${ }^{5}$ It is uncertain why these estimates vary so widely, but one suggestion is that media coverage of the novel symptoms might have influenced people's perception of their symptoms. ${ }^{46}$ Additionally, the selection of patients differed between the studies. Ours and the Icelandic study ${ }^{5}$ included a broad population, including small children and the elderly, who might not be able to report specific symptoms of smell and taste. Finally, patients were not systematically asked about alterations of smell and taste in our study. It is therefore possible that symptoms were present but just not presented. 
Another important finding was that among SARS-CoV-2 positive, $32 \%$ had been in contact with another SARS-CoV-2 infected, which was true for only $8 \%$ of SARS-CoV-2 negative. This makes the question about known contact with a SARS-CoV-2-positive person a key priority in screening of COVID-19 and further in referral to SARS-CoV-2 testing.

Although symptoms including chest pain and irritation/pain in throat were found to be more common among SARS-CoV-2 negative, these symptoms were also relatively common among the positive. Neither of these symptoms can therefore rule out SARS-CoV-2 infection.

\section{Strengths and limitations}

A strength of this study is the stratification of symptoms on age providing important knowledge of age-related differences in symptom presentation of COVID-19. However, we still lack knowledge about whether COVID-19 presents differently depending on other conditions as the occurrence of chronic diseases. Likewise, the timing of calls over the course of illness can vary and reflect the presence of symptoms.

We assessed bias caused by inclusion of calls unrelated to COVID-19 in a sensitivity analysis including only calls where the healthcare professional had suspected COVID-19. This analysis showed similar findings as the main analysis, although the proportion of chest pain among the SARS-CoV-2 negative was smaller in the sensitivity analysis, indicating that some patients with cardiac conditions might have been included in the study population, although they were supposedly only tested due to hospitalisation.

A total of $27 \%$ (662 of 2456) of all SARS-CoV-2 positive in the capital region between 1 April and 20 April $2020^{17} 18$ were identified among calls to the Copenhagen EMS. This study included $12.7 \%$ (312 of 2456) of SARS-CoV-2 positive in the region during the period and $47 \%$ (312 of 662) of those in contact with the Copenhagen EMS. The remaining SARS-CoV-2 positive cases are assumed to be symptomatic healthcare professionals, who could get referred through their employment, symptomatic citizens referred by general practitioners and hospital-admitted patients developing symptoms during admission. Regardless of the type of referral, the symptomatic criteria for being referred to test were the same, also for healthcare professionals. We therefore assume that our results are largely generalisable to the general Danish population.

\section{Implications}

Though symptoms of fever and cough remain common across age groups, other symptoms including loss of smell and taste, headache, nasal congestion and loss of appetite were found to be more age specific. To improve telephone-based screening for COVID-19, differences in symptom presentation across age groups must be considered.

Our findings also emphasised that symptomatic patients, who are aware of possible infection by contact to a SARS-CoV-2-positive individual, must be tested, as these patients are at higher risk of being SARS-CoV-2 positive themselves.

\section{CONCLUSION}

Our study indicates that there are important age-related differences in the symptoms reported by SARS-CoV-2positive patients calling for help or advice at the medical helpline and 1-1-2 emergency number. Loss of smell or taste was found to be indicative of COVID-19, but these symptoms were almost exclusively reported by patients younger than 60 years. Differences in symptom presentation across age groups must be considered in telephonebased screening for COVID-19.

\section{Author affiliations}

${ }^{1}$ Department of Clinical Investigation, Norsjaellands Hospital, Hilleroed, Capital Region, Denmark

${ }^{2}$ Department of Epidemiology and Biostatistics, Aalborg University Hospital, Aalborg, Denmark

${ }^{3}$ Danish Clinical Quality Program (RKKP) National Clinical Registries, Frederiksberg Hospital, Copenhagen, Denmark

${ }^{4}$ Emergency Medical Services Copenhagen, Ballerup, Capital Region, Denmark

${ }^{5}$ Department of Cardiology, Gentofte University Hospital, Hellerup, Denmark

${ }^{6}$ Public Health and Epidemiology Group, Department of Health, Science and Technology, Aalborg University, Aalborg, Denmark

${ }^{7}$ Department of Cardiology, Aalborg University Hospital, Aalborg, Denmark ${ }^{8}$ Department of Obstetrics and Gynaecology, Aalborg University Hospital, Aalborg, Denmark

${ }^{9}$ Department of Cardiology, Nordsjaellands Hospital, Hillerod, Capital Region, Denmark

Contributors Data access and approvals-CT-P, SNFNB and HCC. Initial draft of survey guide-ALM, EHAM, HCC, FG and SNFNB. Data management, analysis and initial manuscript—ALM. Study design, testing and further development of the survey guide, data collection and critically revisiting the manuscript for intellectual content-ALM, EHAM, HCC, FG, SNFNB, NZ, BJ, KBR, MDB, HB and CT-P.

Funding The authors have not declared a specific grant for this research from any funding agency in the public, commercial or not-for-profit sectors.

Competing interests CT-P reports grants from Bayer and Novo Nordisk.

Patient consent for publication Not required.

Ethics approval In accordance with Danish law, no Ethics Committee approval was required for studies of registry data. The study was approved by the Danish Data Protection Agency (record number: P-2019-191).

Provenance and peer review Not commissioned; externally peer reviewed.

Data availability statement By Danish law, the authors are not permitted to share person-level data. Researchers can apply for data access, by acquiring permission from the Danish Data Protection Agency and the Copenhagen Emergency Medical Services in the Capital Region of Denmark.

Supplemental material This content has been supplied by the author(s). It has not been vetted by BMJ Publishing Group Limited (BMJ) and may not have been peer-reviewed. Any opinions or recommendations discussed are solely those of the author(s) and are not endorsed by BMJ. BMJ disclaims all liability and responsibility arising from any reliance placed on the content. Where the content includes any translated material, BMJ does not warrant the accuracy and reliability of the translations (including but not limited to local regulations, clinical guidelines, terminology, drug names and drug dosages), and is not responsible for any error and/or omissions arising from translation and adaptation or otherwise.

Open access This is an open access article distributed in accordance with the Creative Commons Attribution Non Commercial (CC BY-NC 4.0) license, which permits others to distribute, remix, adapt, build upon this work non-commercially, and license their derivative works on different terms, provided the original work is 
properly cited, appropriate credit is given, any changes made indicated, and the use is non-commercial. See: http://creativecommons.org/licenses/by-nc/4.0/.

\section{ORCID iDs}

Amalie Lykkemark Moeller http://orcid.org/0000-0003-2952-2809

Elisabeth Helen Anna Mills http://orcid.org/0000-0002-7499-3880

Stig Nikolaj Fasmer Nikolaj Blomberg http://orcid.org/0000-0002-5073-6820

\section{REFERENCES}

1 Bedford J, Enria D, Giesecke J, et al. COVID-19: towards controlling of a pandemic. Lancet 2020;395:1015-8.

2 Emanuel EJ, Persad G, Upshur R, et al. Fair allocation of scarce medical resources in the time of Covid-19. N Engl J Med 2020;382:2049-55.

3 Spinato G, Fabbris C, Polesel J, et al. Alterations in smell or taste in mildly symptomatic outpatients with SARS-CoV-2 infection. JAMA 2020;323:2089-90.

4 Menni C, Valdes AM, Freidin MB, et al. Real-time tracking of self-reported symptoms to predict potential COVID-19. Nat Med 2020;26:1037-40.

5 Gudbjartsson DF, Helgason A, Jonsson $\mathrm{H}$, et al. Spread of SARSCoV-2 in the Icelandic population. N Engl J Med Overseas Ed 2020;382:2302-15.

6 World Health Organization. Influenza - estimating burden of disease, 2020. Available: http://www.euro.who.int/en/health-topics/ communicable-diseases/influenza/seasonal-influenza/burden-ofinfluenza

7 Lechien JR, Chiesa-Estomba CM, De Siati DR, et al. Olfactory and gustatory dysfunctions as a clinical presentation of mild-to-moderate forms of the coronavirus disease (COVID-19): a multicenter European study. Eur Arch Otorhinolaryngol 2020;277:2251-61.

8 Capital Region of Denmark. Capitial region of Denmark - growth and quality of life, 2017. Available: https://www.regionh.dk/english/about-
the-capital-region/facts-about-the-region/Documents/Pamphlet capital_region_of_denmark_2017_pdfa.pdf

9 Jensen T, Holgersen MG, Jespersen MS, et al. Strategies to handle increased demand in the COVID-19 crisis: a coronavirus EMS support track and a web-based Self-Triage system. Prehospital Emergency Care 2021;25:28-38.

10 Pedersen CB. The Danish civil registration system. Scand J Public Health 2011;39:22-5.

11 Schmidt M, Schmidt SAJ, Adelborg K, et al. The Danish health care system and epidemiological research: from health care contacts to database records. Clin Epidemiol 2019;11:563-91.

12 Randolph JJ. Free-Marginal Multirater Kappa (multirater K [free]): An Alternative to Fleiss' Fixed-Marginal Multirater Kappa. Online submission; Oct 14, 2005.

13 Harris PA, Taylor R, Thielke R, et al. Research electronic data capture (REDCap)--a metadata-driven methodology and workflow process for providing translational research informatics support. J Biomed Inform 2009;42:377-81.

14 Harris PA, Taylor R, Minor BL, et al. The REDCap Consortium: building an international community of software platform partners. $J$ Biomed Inform 2019;95:103208.

$15 \mathrm{R}$ Core Team. R: a language and environment for statistical computing, 2019. Available: https://www.R-project.org/

16 Menni C, Sudre CH, Steves CJ, et al. Quantifying additional COVID-19 symptoms will save lives. Lancet 2020;395:e107-8.

17 COVID-19 i Danmark. Epidemiologisk overvågningsrapport. 01 04-2020, data opdateret kl. 08:00;13 https://files.ssi.dk/covid19/ overvagning/arkiv/covid19-overvaagningsrapport-01042020-apl4

18 COVID-19 i Danmark. Epidemiologisk overvågningsrapport. 204-2020, data opdateret kl. 14.00;17 https://files.ssi.dk/covid19/ overvagning/arkiv/covid19-overvaagningsrapport-20042020-2dd09

19 World Health Organization. Transmission of SARS-CoV-2: implications for infection prevention precautions, 2020. Available: https://www.who.int/news-room/commentaries/detail/ transmission-of-sars-cov-2-implications-for-infection-preventionprecautions 